\title{
Aportación de un caso. Paciente con varicocele y oligozoospermia, con microdeleción en cromosoma $\mathrm{Y}$ : $\mathrm{AZFb}+\mathrm{c}$
}

\author{
Meza-Vázquez HE*, Rosas-Vargas H**, Vite-Velázquez EJ***, De Alba-Mayans AG***. \\ *Sección de Andrología. Departamento de Urología de la UMAE Hospital Especialidades Centro Médico \\ Nacional Siglo XXI. **Unidad de Investigación Médica en Genética Humana de la UMAE Hospital de Pediatría \\ Centro Médico Nacional Siglo XXI. ***UMAE Hospital Centro Médico Nacional La Raza. Méjico.
}

Actas Urol Esp. 2007;31(3):285-288

\section{RESUMEN}

APORTACIÓN DE UN CASO. PACIENTE CON VARICOCELE Y OLIGOZOOSPERMIA, CON MICRODELECIÓN EN CROMOSOMA Y: AZFb+c.

El 13-18\% de las parejas en edad reproductiva llegan a presentar algún tipo de problema de fertilidad, el factor masculino participa en cerca del $50 \%$ de los casos. Las microdeleciones en el cromosoma Yq ocupan sólo el $7,6 \%$ y se relacionan en forma importante con oligozoospermia severa $(<5$ millones $\mathrm{x}$ mililitro OMS 1999) (2-5\%) y azoospermia (5-10\%).

La estructura de la región AZF de Yq se divide en AZFa, AZFb, AZFc y AZFd. Las microdeleciones pueden ser simples o combinadas, siendo la microdelección AZFc (59-65\%), la más común.

En casos con oligozoospermia severa (o azoospermia) se sugiere estudio de microdeleciones en cromosoma Y, independientemente de las enfermedades concomitantes (ejemplo varicocele), como parte del pronóstico de fertilidad.

ICSI con selección de sexo, sería el tratamiento adecuado en estos casos, se encuentra en fase de estudio. Palabras clave: Microdeleciones Yq. Azoospermia. Oligozoospermia. Infertilidad masculina.

\section{ABSTRACT}

CASE REPORT. PATIENT WITH VARICOCELE \& OLIGOZOOSPERMIA WITH Y CHROMOSOME MICRODELETION: AZFb+c.

The 13-18\% of the couples in reproductive age gets to present some kind of fertility problem, the male factor participates in near 50\% of the cases. The microdeletions in the Yq chromosome occupy single the 7,6\%; severe oligozoospermia and azoospermia are related with these alterations. The structure of region AZF of Yq is divided in AZFa, AZFb, AZFc and AZFd. The microdeletions can be simple or combined, the microdeletion AZFc (59-65\%) is the commonest. In cases with severe oligozoospermia (or azoospermia), study of microdeletions in Y chromosome is suggested. ICSI with selection of sex, would be the treatment adapted in these cases, is in phase of study.

Keywords: Yq microdeletions. Azoospermia. Oligozoospermia. Infertility.

$\mathrm{E}$ 13-18\% de las parejas en edad reproductiva llegan a presentar algún problema de fertilidad $^{1,2}$. El factor masculino se ha identificado en cerca de la mitad de los casos y como factor solitario se observa en $20-30 \%$ de los $\operatorname{casos}^{2,3}$. No se reconoce causa aparente (idiopática) en el 30\% de los casos de infertilidad masculina ${ }^{4}$. Dentro de las causas de infertilidad masculina de origen genético, las microdeleciones en Yq (brazo largo del cromosoma Y) ocurre en aproximadamente $7,6 \%$ de los casos ${ }^{1}$.

El cromosoma $\mathrm{Y}$ es indispensable para que tengan lugar los primeros acontecimientos de la determinación del sexo y espermatogénesis normal. El paso inicial del desarrollo sexual (determinación testicular) normal del varón exige la 
expresión del gen SRY, pero para la espermatogénesis normal se necesita la expresión de otros genes del cromosoma $\mathrm{Y}^{5,6}$. Tiepolo y Zuffardi, fueron los primeros en advertir grandes deleciones de la región distal de cromosoma Yq en un grupo de varones con azoospermia a lo que denominaron como región del factor de azoospermia (AZF) (azoospermia factor) ${ }^{7}$.

La prevalencia de microdeleciones de cromosoma $\mathrm{Y}$ en pacientes con azoospermia no obstructiva se encuentra entre 5-10\%,en pacientes con oligozoospermia severa se observa entre 2$5 \% 3,8$. En otros estudios, las microdeleciones en oligozoospermia severa alcanza hasta $14,1 \%$, la detección varia ampliamente de acuerdo a la metodología empleada y a criterios de inclusión y exclusión de los pacientes ${ }^{9}$.

En la estructura de la región AZF de Yq se identifican cuatro regiones. AZFa , AZFb, AZFc y AZFd (esta última, se cree localizada entre las regiones $\mathrm{AZFb}$ y $\mathrm{AZFc})^{10}$ (Fig. 1).

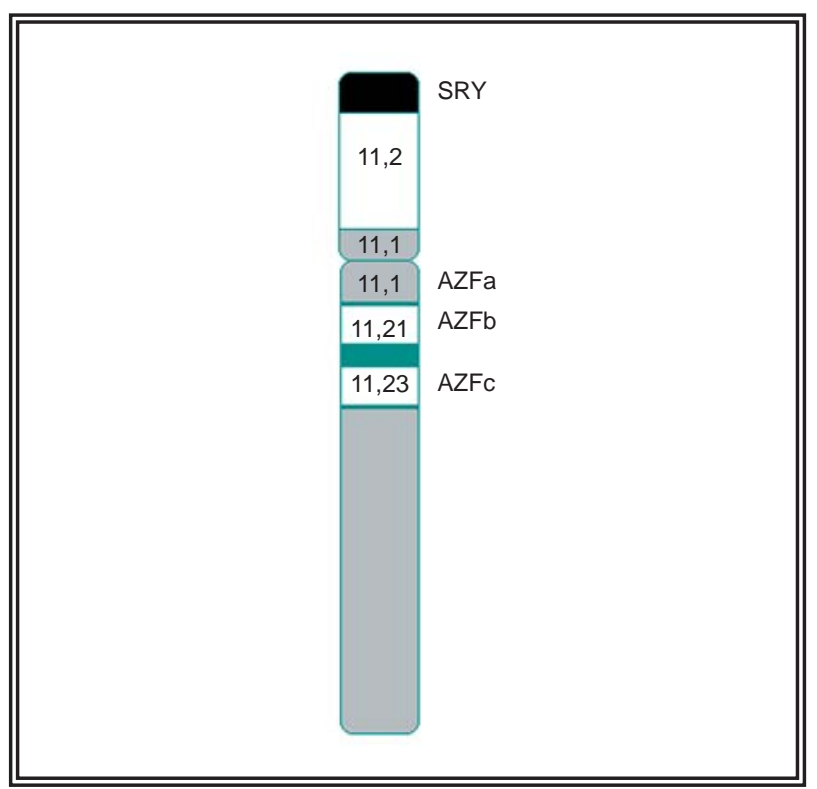

FIGURA 1. Esquema gráfico del cromosoma Y, mostrando las regiones $A Z F a, A Z F b$ y $A Z F c$.

Según Foresta y cols. la prevalencia relativa de deleciones en la región $\mathrm{AZF}$ es para $\mathrm{AZFa}=$ $4,9 \%, \mathrm{AZFb}=15,8 \%, \mathrm{AZFc}=59,6 \%, \mathrm{AZF} \mathrm{a}+\mathrm{b}=$ $1,5 \%, A Z F \mathrm{~b}+\mathrm{c}=8,3 \%, \mathrm{AZF} \mathrm{a}+\mathrm{b}+\mathrm{c}=3,8 \%$ y fuera de AZF $6 \%{ }^{10}$.

En el estudio de Foresta, la prevalencia relativa de microdeleción $\mathrm{AZFb}+\mathrm{c}$ es de $8,3 \%$, del total de microdeleciones Yq, y de estos 96\% son azoospermicos y $4 \%$ presentan oligozoospermia severa $^{10}$.

\section{CASO CLÍNICO}

Varón de 24 años sin enfermedades concomitantes.

Antecedentes andrológicos: Descenso testicular al nacimiento, no circuncidado, aparición de caracteres sexuales secundarios normales a los 14 años, espermarquia a los 14 años, inicio de vida sexual a los 16 años, dos parejas sexuales, sin antecedentes de enfermedades de transmisión sexual. Erecciones matutinas 4/7, sin problemas para la erección voluntaria, libido conservada, sin problemas eyaculatorios.

Exploración fisica: Fenotipo masculino sin fascies característica, sin alteraciones cardiopulmonares, mamas normales acorde con edad y sexo. Distribución de vello androide, pene con prepucio retráctil, meato central, testículo izquierdo con volumen disminuido en comparación a testículo contralateral, de consistencia disminuida y varicocele grado III; testículo derecho de consistencia normal sin evidencia clínica de varicocele.

Padecimiento actual: infertilidad de 1 año y medio de evolución, pareja femenina de 23 años, con protocolo de estudio de fertilidad sin alteraciones.

Laboratorio: Biometría hemática: hemoglobina $15,3 \mathrm{~g} / \mathrm{dl}$, leucocitos 8,000 cél/ $\mu$, TP 13,8 segundos, TTP 28,9 segundos.

Buímica sanguinea: glucosa $80 \mathrm{mg} / \mathrm{dl}$, creatinina $0,96 \mathrm{mg} / \mathrm{dl}$, sodio $142 \mathrm{meq} / \mathrm{L}$, potasio 4,4 $\mathrm{meq} / \mathrm{L}$, cloro $103 \mathrm{meq} / \mathrm{L}$.

Examen general de orina: $\mathrm{pH}$ 6, densidad 1,020, leucocitos 2-4 por campo.

Hormonas: LH 5,2 (0.8-7.6 mUI/ml) FSH 12,2 $(0,7-11,1 \mathrm{mUI} / \mathrm{ml})$, oestradiol $12,4(0-56 \mathrm{pg} / \mathrm{ml})$ testosterona $588 \mathrm{ng} / \mathrm{dl}$ (270-1734).

Cultivos de orina y semen negativos: Búsqueda de Chlamydia, Micoplasma y Ureaplasma en secreción uretral negativos.

Análisis de semen: Aspecto normal, licuefacción normal, viscosidad normal, volumen $3 \mathrm{ml}$, $\mathrm{pH}$ 8,0, concentración 3 millones/ml, motilidad tipo $\mathrm{d}=100 \%$

Morfología (Kruger): normales 0\%, cola anormal $28 \%$, amorfos $36 \%$, acintados $28 \%$, alfiler $7 \%$, duplicados $1 \%$. 
Oligozoospermia, astenozoospermia, y teratozoospermia severa.

Ultrasonido escrotal: Testículo izquierdo de 4,68 cc con varicocele, testículo derecho 10,92 cc, ambos epidídimos normales.

Cariotipo: 46,XY.

Microdeleciones cromosoma Y mediante técnica de reacción en cadena polimerasa múltiplex de STS cromosoma Y :

Múltiplex A:

ZFY, SRY, sY84 (AZFa) presentes.

sY254 (AZFc), sY127 (AZFb) ausentes.

Múltiplex B:

ZFY, SRY, sY86(AZFa) presentes

sY134 (AZFb), sY255 (AZFc) ausentes.

Los análisis se realizaron por triplicado $\mathrm{y}$ como referencia se tomaron controles positivos y negativos.

\section{DISCUSIÓN}

Habitualmente en estos casos, un varón con infertilidad y varicocele grado III se propondría realizar varicocelectomía y esperar recuperación de parámetros seminales (un año). Actualmente, en aquellos pacientes con infertilidad y oligozoospermia severa (o azoospermia) por análisis de semen, se sugiere solicitar pruebas de detección de microdeleciones Yq. A pesar de la presencia de otras entidades concomitantes que puedan provocar daño testicular, tal como el varicocele o criptorquidea $^{9}$.

El presente caso es de interés debido a la baja prevalencia relativa de las microdeleciones AZF $\mathrm{b}+\mathrm{c}(8,3 \%)$ con oligozoospermia severa $(4 \%)$ (es más común la presencia de azoospermia) ${ }^{9}$.

En este caso se solicitó, en base a la cuenta de espermatozoides (3 millones $/ \mathrm{ml}$ ) $\mathrm{PCR}$ para microdeleciones cromosoma $\mathrm{Y}$, antes de continuar con protocolo de infertilidad para ofrecer un pronóstico genético, con los resultados arriba descritos (Fig. 2).

$\mathrm{El}$ tener microdeleciones $\mathrm{Yq} \mathrm{AZFb}+\mathrm{c}$ tiene mal pronóstico reproductivo, no existen estrategias para incrementar la cuenta espermática por ser un problema genético de origen.

Los pacientes con microdeleciones Yq son candidatos a reproducción asistida con ICSI y selección de sexo, actualmente en investigación ${ }^{1}$; es demostrado que los recién nacidos masculinos

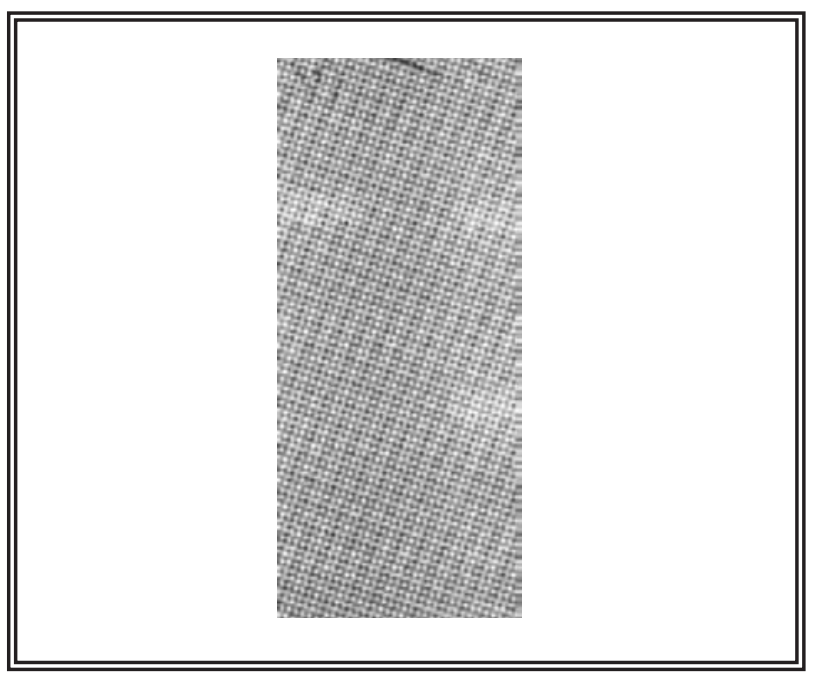

FIGURA 2. PCR de cromosoma Y con microdeleciones $\mathrm{Yq}$ donde no se aprecian bandas correspondientes a STS.

heredarán la microdeleción Yq del padre en el $100 \%$ de los casos. En casos, que si se han sometido a ICSI, la longitud de la microdeleción no ha aumentado y se espera que presenten la misma capacidad espermatogénica del padre. Tanto las hijas como los hijos son somáticamente sanos ${ }^{11}$.

Por otro lado, no existe evidencia de relación directa del varicocele con las microdeleciones Yq y parecen ser sólo patologías concomitantes ${ }^{9,11}$. Por lo que se deberá abordar el varicocele como parte integral del manejo de este paciente, asesorando genéticamente y ofreciendo un pronóstico (Fig. 3).

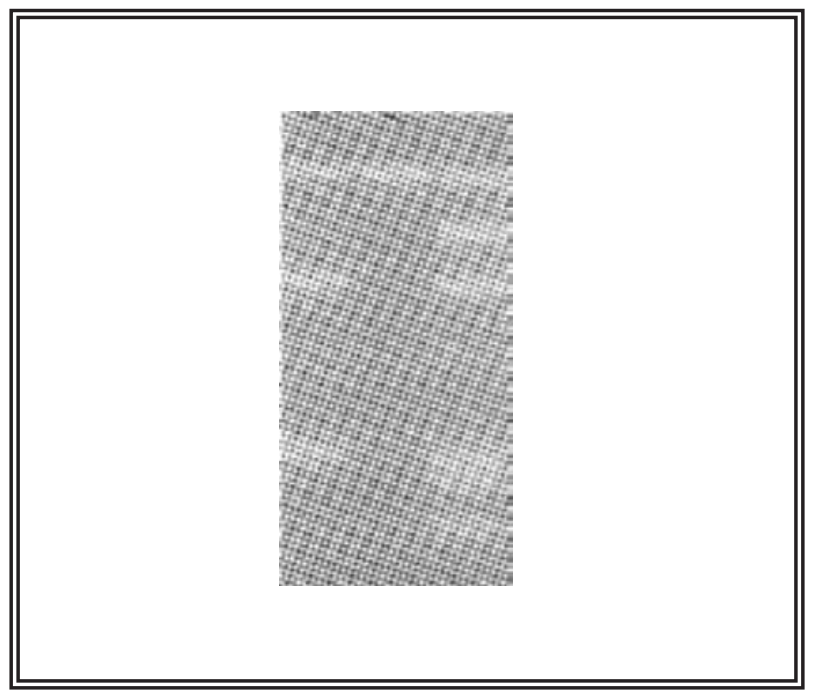

FIGURA 3. PCR de cromosoma Y sin alteraciones donde se aprecian bandas completas de STS. 

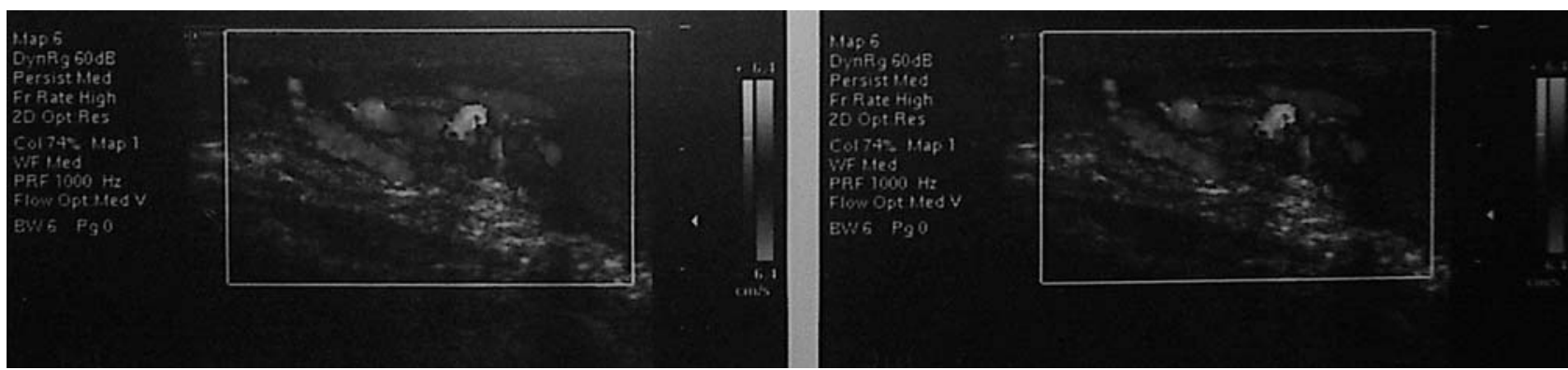

FIGURA 4. Ultrasonido doppler testicular izquierdo, basal y con Valsalva, se aprecia dilatación venosa en ambas fases (varicocele).

\section{CONCLUSIONES}

De acuerdo a los autores revisados, se sugiere que en aquellos pacientes con infertilidad primaria más oligozoospermia severa (o azoospermia), solicitar la detección de microdeleciones de cromosoma Y como parte del estudio inicial de infertilidad, independientemente de las patologias concomitantes que puedan estar presentes, como es el varicocele en este paciente (Fig. 4).

El manejo debe ser multidisciplinario con el apoyo de genética clínica, urología y andrología para ofrecer una mejor asesoría a este tipo de pacientes, y en caso de someterse a ICSI, advertir el riesgo de heredar genéticamente la enfermedad.

\section{REFERENCIAS}

1. Stouffs K, Lissens W, Tournaye H, Van Steirteghem A, Liebaers I. The choice and outcome of the fertility treatment of 38 couples in whom the male partner has a Yq microdeletion. Hum Reprod. 2005;20(7):1887-1896.

2. Wong WY, Thomas CM, Merkus JM, Zielhuis GA, SteegersTheunissen RP. Male factor subfertility: possible causes and the impact of nutritional factors. Fertil Steril. 2000;73(3): 435-441.

3. Nieschlag E., Behre H., Mesched., et al . Chapter 8 Disoders at the testicular level in Andrology Male Reproductive Health and Dysfunction. $2^{\text {nd }}$ edition Springer-Verlag Berlin Heidelberg. 2001;143-176.

4. Peterlin B, Kunej T, Sinkovec J, Gligorievska N, Zorn B. Screening Y chromosome microdeletions in 226 Slovenian subfertile men. Hum Reprod. 2002;17(1):17-24.
5. Pagani R, Brugh VM 3rd, Lamb DJ. Genes of Y-Chromosome: Male Infertility. Urol Clin North Am. 2002; 29(4): 751-759.

6. Ferlin A, Tessari A, Ganz F, Marchina E, Barlati S, Garolla A, et al. Association of partial AZFc region deletions with spermatogenic impairment and male infertility. J Med Genet. 2005;423(3):209-213.

7. Tiepolo L., Zuffardi O. Localization of factors controlling spermatogenesis in the nonfluorescent portion of the human Y chromosome long arm. Hum Genet. 1976;34(2): 119-124.

8. Simoni M, Gromoll J, Dworniczak B, Rolf C, Abshagen K, Kamischke A, et al. Screening for deletions of the Y chromosome involving the DAZ (Deleted in Azoospermia) gene in azoospermia and severe oligozoospermia. Fertil Steril. 1997;67(3):542-547.

9. Foresta C, Moro E, Ferlin A. Y Chromosome Microdeletions and Alterations of Spermatogenesis. Endocrine Rev. 2001; 22(2):226-239.

10. Kent-First M, Muallem A, Shultz J, Pryor J, Roberts K, Nolten W, et al. Defining regions of the Y chromosome responsible for male infertility and identification of a fourth AZF region (AZFd) by Y chromosome microdeletion detection. Mol Reprod Dev. 1999;53(1):27-41.

11. Oates RD, Silber S, Brown LG, Page DC. Clinical characterization of 42 oligospermic or azoospermic men with microdeletion of the AZFc region of the chromosome, and of 18 children conceived via ICSI. Hum Reprod. 2002; 17 (11):2813-2824.

Correspondencia autor: Dr. HE Meza Vázquez. Sección de Andrologia, Departamento de Urología de la UMAE

Hospital Especialidades Centro Médico Nacional Siglo XXI y la Unidad de Investigación Médica en Genética Humana de la UMAE Hospital de Pediatría Centro Médico Nacional Siglo XXI. Méjico E-mail autor: hemezavzq@yahoo.com.mx

Información artículo: Nota clínica

Trabajo recibido: noviembre 2005

Trabajo aceptado: febrero 2006 